\title{
Interaction of thermal contact resistance and frictional heating in thermoelastic instability
}

\author{
M. Ciavarella a, L. Johansson ${ }^{\text {b }}$, L. Afferrante ${ }^{\text {a }}$, A. Klarbring ${ }^{\text {b }}$, J.R. Barber ${ }^{\text {c,* }}$ \\ ${ }^{\text {a }}$ CEMEC-PoliBA-Centre of Excellence in Computational Mechanics, V.le Japigia 182, \\ Politecnico di Bari, 70125 Bari, Italy \\ ${ }^{\mathrm{b}}$ Division of Mechanics, Department of Mechanical Engineering, Linköping University, S-581 83, Linköping, Sweden \\ ${ }^{\mathrm{c}}$ Department of Mechanical Engineering, University of Michigan, 2250 G. G. Brown Building 2350 Hayward Street, Ann Arbor, \\ MI 48109-2125, USA
}

Received 23 May 2003; received in revised form 23 May 2003

\begin{abstract}
Thermoelastic contact problems can posess non-unique and/or unstable steady-state solutions if there is frictional heating or if there is a pressure-dependent thermal contact resistance at the interface. These two effects have been extensively studied in isolation, but their possible interaction has never been investigated. In this paper, we consider an idealized problem in which a thermoelastic rod slides against a rigid plane with both frictional heating and a contact resistance. For sufficiently low sliding speeds, the results are qualitatively similar to those with no sliding. In particular, there is always an odd number of steady-state solutions; if the steady-state is unique it is stable and if it is non-unique, stable and unstable solutions alternate, with the outlying solutions being stable. However, we identify a sliding speed $V_{0}$ above which the number of steady states is always even (including zero, implying possible non-existence of a steadystate) and again stable and unstable states alternate. A parallel numerical study shows that for $V>V_{0}$ there are some initial conditions from which the contact pressure grows without limit in time, whereas for $V<V_{0}$ the system will always tend to one of the stable steady states.
\end{abstract}

(c) 2003 Elsevier Ltd. All rights reserved.

Keywords: Thermoelastic contact; Thermoelastic instability (TEI); Thermal contact resistance; Uniqueness

\section{Introduction}

Contact problems for thermoelastic bodies can exhibit instability associated with thermomechanical coupling at the contact interface. Two distinct categories of thermomechanical coupling can be identified.

In static thermoelastic contact, the surface roughness of the contacting bodies will generally cause a local pressure-dependent thermal contact resistance, resulting in coupling between the elastic problem and the heat conduction problem (Barber et al., 1980). This can cause instability and erratic behaviour in heat

\footnotetext{
${ }^{*}$ Corresponding author. Tel.: +1-7349-360406; fax: +1-7346-156647.

E-mail address: jbarber@umich.edu (J.R. Barber).
} 
exchanger systems involving contacting interfaces (Srinivasan and France, 1985). Instability occurs when the temperature difference or the heat flux between the contacting bodies exceeds a certain critical value (Yeo and Barber, 1994). The most common form of instability is associated with heat flow into the more distortive material, where the distortivity

$$
\delta=\frac{\alpha(1+v)}{K}
$$

and $\alpha, v, K$ are the coefficient of thermal expansion, Poisson's ratio and thermal conductivity, respectively. Static thermoelastic contact problems can also exhibit multiple steady-state solutions for this direction of heat flow and in some simple systems the criteria for stability and uniqueness of the steady-state have been shown to be identical (Barber et al., 1980; Barber, 1986). In such cases, disturbance of the system from an unstable steady-state will cause it to gravitate towards an alternative stable state. However, for some material combinations, instability can occur for sufficiently high heat fluxes in both directions (Zhang and Barber, 1990) and unique steady states can be unstable (Zhang and Barber, 1993).

A different category of thermoelastic contact instability can occur if there is sliding between the contacting bodies, in which case the work done against frictional tractions leads to localized heating proportional to the local contact pressure. Instabilities due to this source are found in energy dissipating systems such as brakes and clutches and are known as frictionally-excited thermoelastic instability (TEI) (Barber, 1969). The thermomechanical coupling in this case is proportional to the sliding speed $V$ and for a given system there is a critical sliding speed $V_{\mathrm{c}}$ above which the system is unstable (Dow and Burton, 1972). If the total contact force is prescribed and there is no wear, the system will then tend to an alternate stable steady-state involving a reduced contact area (Burton et al., 1973; Zagrodzki et al., 2001). However, if the displacement is constrained, as in a shaft rotating in a bearing, the pressure may grow without limit, causing seizure of the system (Burton and Staph, 1967; Tu and Stein, 1995).

In real sliding systems, both effects will be simultaneously present. There will be frictional heating, but also the surfaces are rough and hence there will be some resistance to heat flow across the interface. In these circumstances, both categories of TEI might be anticipated, depending on the sliding velocity and the relative magnitude of the temperature difference between the bodies. However, it is not clear whether the resulting instabilities will be distinct or whether a single criterion will apply involving all the thermomechanical parameters of the system. In the present paper, we shall investigate this issue in the context of a very simple model - the sliding of a one-dimensional rod against a rigid wall, with frictional heating and a pressure-dependent thermal contact resistance.

\section{The rod model}

Fig. 1 shows a thermoelastic $\operatorname{rod} A C$ of length $L$, built in to a rigid wall at $A$ and separated from a second rigid wall $B$ by a small gap $g$. If the rod is heated sufficiently to make contact with the wall at $B$, the gap will close and a contact pressure $p$ will be established. The wall at $B$ is assumed to be moving at speed $V$ perpendicular to the figure, so in the case of contact there will be frictional heating $q$ per unit area given by

$$
q=f V p
$$

where $f$ is the coefficient of friction. We also suppose that there exists thermal resistance $R$ to heat flow between $C$ and $B$ that depends on the magnitude of the gap and the contact pressure.

In defining appropriate thermal boundary conditions for this case, it is essential to define the relative location of the heat source and the thermal resistance (Barber, 1967, 1970; Johansson, 1993). In practical cases, this will depend on the nature of the sliding materials. For example, if there is a significant difference in hardness between the materials, we would expect the frictional energy dissipation to arise largely as a 


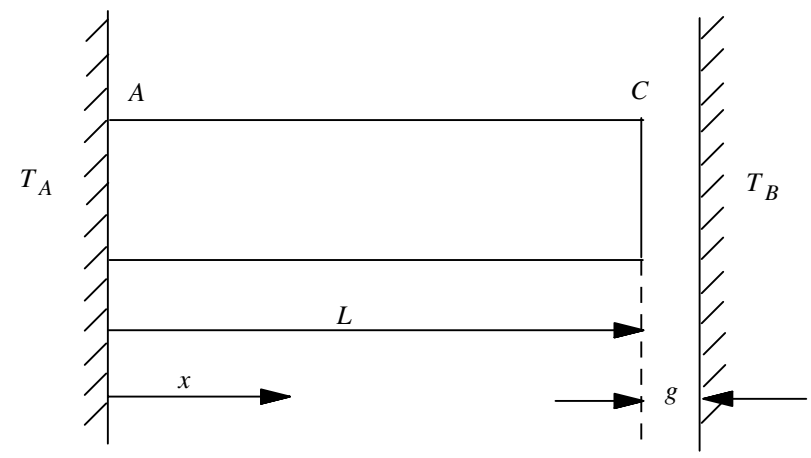

Fig. 1. The rod model.

result of plastic deformation in the softer material, which will therefore be the source of most of the heating. In the present case, we shall assume that the frictional heat source is located at $C$ and hence that any heat flow to the wall has to pass through the resistance $R$.

With this assumption, the thermal boundary conditions at $C$ can be written

$$
\begin{aligned}
& q_{1}=\frac{T_{C}-T_{B}}{R}, \\
& q_{2}=K \frac{\mathrm{d} T}{\mathrm{~d} x}(L), \\
& q_{1}+q_{2}=f V p,
\end{aligned}
$$

where $q_{1}, q_{2}$ are the heat flow into the wall and into the rod respectively and $T$ is temperature. We also note that the temperature in the rod must satisfy the heat conduction equation

$$
\frac{\partial^{2} T}{\partial x^{2}}=\frac{1}{k} \frac{\partial T}{\partial t}
$$

where $k$ is the thermal diffusivity of the rod material and $t$ is time.

The unrestrained thermal expansion of the rod is

$$
\Delta=\alpha \int_{0}^{L} T(x) \mathrm{d} x
$$

and if this is insufficient to close the gap, we shall have

$$
g=g_{0}-\Delta,
$$

where $g_{0}$ is defined as the gap when $T(x)=0$ for all $x$. If $\Delta>g_{0}$, the rod will contact the wall and elementary calculations show that the resulting contact pressure, assuming quasi-static conditions, will be given by

$$
-\frac{p L}{E}=g_{0}-\Delta,
$$

where $E$ is Young's modulus for the rod material. Following Barber et al. (1980), it is convenient to regard Eq. (8) as defining a 'generalized' gap function $g$ such that

$$
g=-\frac{p L}{E} \quad \text { if } p>0 .
$$


With this notation, the same equations apply for both contact and separation, except that the term $f V p$ in (5) must be set to zero when $g>0$.

Eqs. (3)-(9), including the inequality constraints, completely define the transient behaviour of the system, where the resistance $R$ is assumed to be a continuous function of $g$. The same equations must therefore define both the steady-state solutions and their stability.

\section{The steady-state problem}

We first investigate the possible steady states of the system. In particular, we seek to establish conditions under which a steady-state exists and is unique. In the steady-state, Eq. (6) has only the linear solution

$$
T(x)=\frac{T_{A} x+T_{C}(L-x)}{L}
$$

and hence

$$
\Delta=\frac{\alpha L\left(T_{A}+T_{C}\right)}{2}
$$

from (7).

Eliminating $q_{1}, q_{2}$ from Eqs. (3)-(5) and using (11), we obtain

$$
\frac{T_{C}-T_{B}}{R}=f V p+\frac{K\left(T_{A}-T_{C}\right)}{L}
$$

from which

$$
T_{C}=\frac{K R T_{A}+L T_{B}}{(L+K R)}+\frac{f V p R L}{(L+K R)}
$$

and hence

$$
\Delta=\alpha L T_{A}+\frac{\alpha L^{2} f V p R}{2(K R+L)}-\frac{\alpha L^{2}\left(T_{A}-T_{B}\right)}{2(K R+L)}
$$

from (12).

Using this result, Eq. (8) and the associated inequalities can be combined in the equation

$$
\mathscr{F}(g) \equiv g-\left(g_{0}-\alpha L T_{A}\right)-\frac{\widehat{V} K g H(-g) R(g)}{(K R(g)+L)}-\frac{\alpha L^{2}\left(T_{A}-T_{B}\right)}{2(K R(g)+L)}=0,
$$

where

$$
\widehat{V}=\frac{f V E \alpha L}{2 K}
$$

is a dimensionless sliding speed and $H(g)$ is the Heaviside step function.

\section{Stability of the steady-state}

Eq. (16) defines a function $\mathscr{F}(g)$ that must be zero at any steady-state of the system. Notice that the function is continuous, since the term containing the Heaviside function is zero at $g=0$. 
The stability of the corresponding steady states can be investigated by determining the conditions under which a small temperature perturbation can grow exponentially in time. We first note that the heat flow out of the rod at the end $C$ is

$$
q \equiv-q_{2}=\frac{T_{C}-T_{B}}{R}-f V p
$$

from (3), (5) and hence, for small perturbations $\Delta q, \Delta T_{C}, \Delta p$ about a steady-state,

$$
\Delta q=\frac{\Delta T_{C}}{R}-\frac{\left(T_{C}-T_{B}\right) R^{\prime}}{R^{2}} \Delta g-f V \Delta p,
$$

where the quantities $R, T_{C}, p$ define the steady-state conditions.

Substituting (14) into (19) and using

$$
\Delta p=-\frac{E \Delta g}{L},
$$

we obtain

$$
\Delta q=\frac{\Delta T_{C}}{R}-\frac{K\left(T_{A}-T_{B}\right) R^{\prime}}{R(L+K R)} \Delta g-\frac{f V p L R^{\prime}}{R(L+K R)} \Delta g+\frac{f V E}{L} \Delta g .
$$

For transient perturbations of the form $T(x, t)=\Theta(x) \exp (b t)$, Barber et al. (1980) showed that

$$
\begin{aligned}
& \Delta q=-B K \lambda \cosh (\lambda L) \mathrm{e}^{b t}, \\
& \Delta T_{C}=B \sinh (\lambda L) \mathrm{e}^{b t}, \\
& \Delta g=-\frac{B \alpha}{\lambda} \mathrm{e}^{b t}[\cosh (\lambda L)-1],
\end{aligned}
$$

where $\lambda=\sqrt{b / L}$ and $B$ is an arbitrary constant.

Substituting (22)-(24) into (21), using

$$
p=-\frac{E g}{L} ; \quad z=\lambda L
$$

and multiplying by $z$, we obtain

$$
\frac{K}{L} z^{2} \cosh (z)+\frac{1}{L} z \sinh (z)+\left[\frac{\alpha K L\left(T_{A}-T_{B}\right) R^{\prime}}{R(L+K R)}-\frac{f V L E \alpha g R^{\prime}}{R(L+K R)}-f V E \alpha\right](\cosh (z)-1)=0 .
$$

This equation is of the form

$$
C_{1} z^{2} \cosh (z)+C_{2} z \sinh (z)+C_{3}(\cosh (z)-1)=0
$$

with $C_{1}>0$ and $C_{2}>0$. Under these conditions, Barber et al. (1980) showed that all the zeros of (27) will satisfy the condition $\mathfrak{R}(b)<0$ (indicating stability), if and only if

$$
2 C_{1}+2 C_{2}+C_{3}>0 .
$$

In the present case, this leads to the stability criterion

$$
\frac{2 K}{L}+\frac{2}{L}+\frac{\alpha K L\left(T_{A}-T_{B}\right) R^{\prime}}{R(L+K R)}-\frac{f V L E \alpha g R^{\prime}}{R(L+K R)}-f V E \alpha>0
$$

or, after multiplying by the positive constant $R L / 2(L+K R)$ and using (17), 


$$
1+\frac{\alpha K L^{2}\left(T_{A}-T_{B}\right) R^{\prime}}{2(L+K R)^{2}}-\frac{\widehat{V} K L g R^{\prime}}{(L+K R)}-\frac{\widehat{V} K R}{(L+K R)}>0 .
$$

Comparison with Eq. (16) shows that (30) can be written

$$
\frac{\mathrm{d} \mathscr{F}}{\mathrm{d} g}>0
$$

In this proof, we have tacitly assumed that there is contact $(p>0, g<0)$ so that $H(-g)=1$. If $g>0$, all the equations are modified by omitting the terms involving $V$, which is equivalent to setting $\widehat{V}=0$ in (16), (30), leaving the conclusion unchanged. The presence of the step function implies that $\mathscr{F}$ is undifferentiable at the transition $g=0$ between contact and separation. In the special case where a steady-state occurs at this point, its stability can be determined by examining the signs of the left and right derivatives of $\mathscr{F}$.

\section{Existence, uniqueness and stability}

Some insight into the existence and stability of solutions can be gained by considering the behaviour of $\mathscr{F}$ as $g \rightarrow \pm \infty$.

The resistance function $R(g)$ must be a positive function of $g$ and hence $\mathscr{F} \rightarrow \infty$ as $g \rightarrow \infty$. However, at the opposite extreme $g \rightarrow-\infty$, we have

$$
\mathscr{F} \rightarrow\left(1-\frac{\widehat{V} K R_{\infty}}{\left(K R_{\infty}+L\right)}\right) g-\left(g_{0}-\alpha L T_{A}\right)-\frac{\alpha L^{2}\left(T_{A}-T_{B}\right)}{2\left(K R_{\infty}+L\right)},
$$

where

$$
R_{\infty}=\lim _{g \rightarrow-\infty} R(g) .
$$

It follows that if

$$
\widehat{V}<\widehat{V}_{0} \equiv 1+\frac{L}{K R_{\infty}},
$$

we shall have $\mathscr{F} \rightarrow-\infty$ as $g \rightarrow-\infty$. In this case, $\mathscr{F}$ is a continuous function of $g$ extending from $+\infty$ to $-\infty$ and hence (16) must have an odd number of roots (except in the case of repeated roots) and in particular, at least one root, thus establishing an existence theorem for the system for $\widehat{V}<\widehat{V}_{0}$.

Furthermore, since $\mathscr{F}$ has to cross the $g$ axis from the negative to the positive side with increasing $g$, we conclude from (31) that when the steady-state is unique it must also be stable. If there is an odd number of steady states, they must be alternately stable and unstable, the outermost states being stable. This conclusion agrees with that of Barber et al. (1980) for the system without frictional heating $(\widehat{V}=0)$. Thus, the inclusion of frictional heating does not cause a qualitative difference in the system behaviour for sufficiently small sliding speed, defined by (34). Notice also that condition (34) holds for all sliding speeds if $R_{\infty}=0$.

If $\widehat{V}>\widehat{V}_{0}, \mathscr{F}$ will tend to $+\infty$ at both extremes and the number of roots of (16) must be even or zero. Thus, there is no guarantee of existence of a steady-state in this case. If some steady states exist, they must be alternately stable and unstable, with the solution at the largest value of $g$ being stable, since the condition $\mathscr{F} \rightarrow \infty$ as $g \rightarrow \infty$ implies that the last zero crossing occurs from the negative to the positive side with increasing $g$. Thus, the solution at the smallest value of $g$ (or the largest contact pressure) must be unstable. This raises the question as to what will be the transient behaviour of the system if it is perturbed from this unstable steady-state? Clearly, for certain perturbations it will gravitate towards the stable steady-state, but 
if the sign of the perturbation is changed, we might expect a state in which the contact pressure increases without limit. This is also the probable outcome in cases where there is no steady-state.

\section{A special case}

To explore these questions more thoroughly, we consider the special case where

$$
\begin{aligned}
R(g) & =B-\frac{A}{g} \quad \text { if } g<0 \\
& =\infty \quad \text { if } g>0 .
\end{aligned}
$$

In other words, no heat flow occurs between the rod and the wall when there is a gap, however small, and the resistance to heat flow during contact contains a constant term and a term which is inversely proportional to contact pressure. This is a reasonable approximation to experimental observations and theoretical predictions of thermal contact resistance (Cooper et al., 1969; Shlykov and Ganin, 1964). In this case, $R_{\infty}=B>0$ and we anticipate problems of non-existence of the steady-state at sufficiently high sliding speeds. An alternative interpretation of Eq. (35) is that the term $A / g$ represents the resistance between the rod and the wall associated with the roughness of the contacting surfaces, whilst the constant term $B$ represents the resistance to heat flow from the wall to the environment.

Substituting (35) into (16), we obtain (after some algebra)

$$
g-\left(g_{0}-\alpha L T_{A}\right)=\frac{\alpha L\left(T_{A}-T_{B}\right) f(g)}{2}+\frac{\widehat{V} K H(-g)(B g-A f(g))}{(L+K B)},
$$

where

$$
\begin{array}{rlr}
f(g) & =\frac{L}{K R(g)+L}=0 & \text { if } g>0 \\
& =\frac{L g}{L g+K B g-K A} & \text { if } g<0
\end{array}
$$

is a continuous monotonic function of $g$ which tends to a maximum value of $1 /(1+B K / L)$ as $g \rightarrow-\infty$.

Eq. (36) can be rearranged as

$$
\begin{aligned}
f(g) & =F_{\mathrm{c}}(g) \quad \text { if } g<0 \\
& =F_{s}(g) \quad \text { if } g>0,
\end{aligned}
$$

where

$$
\begin{aligned}
& F_{\mathrm{c}}(g)=\left(g\left(1-\frac{\widehat{V} K B}{(L+K B)}\right)-\left(g_{0}-\alpha L T_{A}\right)\right) /\left(\frac{\alpha L\left(T_{A}-T_{B}\right)}{2}-\frac{\widehat{V} K A}{(L+K B)}\right) \\
& F_{S}(g)=\frac{2 g-2\left(g_{0}-\alpha L T_{A}\right)}{\alpha L\left(T_{A}-T_{B}\right)}
\end{aligned}
$$

showing that steady-state solutions for the system are defined by intersections between the function $f(g)$ of (37) and separate straight line segments in the regions $g<0$ and $g>0$.

A graphical interpretation of the solution of Eqs. (38), (39) is shown in Fig. 2(a-d). The left hand side of these equations is represented by the curve $f(g)$ in the range $g<0$ and by the $g$-axis in $g>0$, whilst the right hand side is represented by the two line straight line segments $F_{\mathrm{c}}(g), F_{s}(g)$ of (40), (41). 


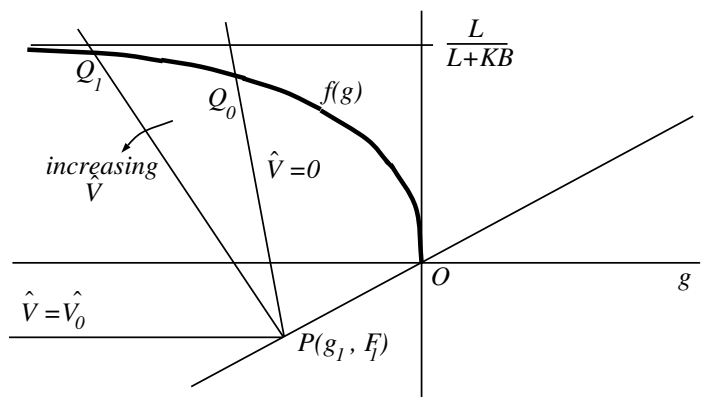

(a)

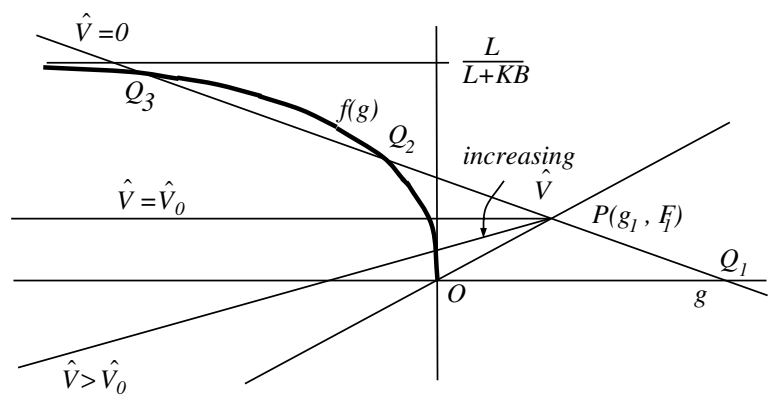

(c)

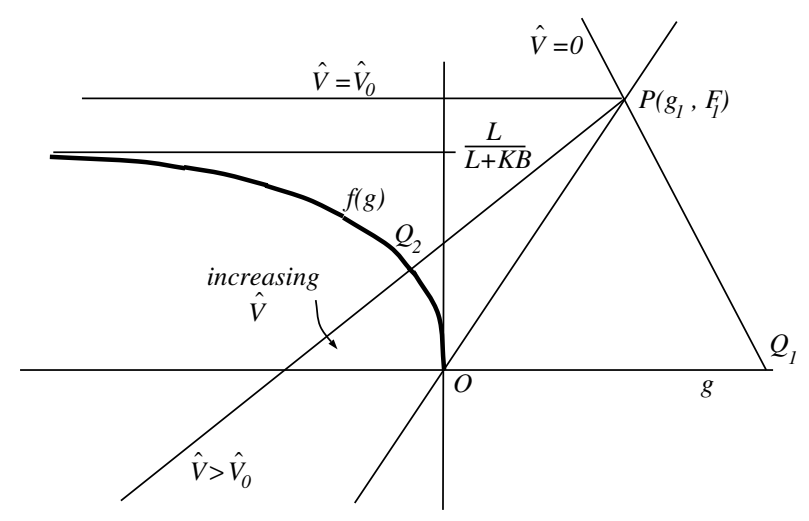

(b)

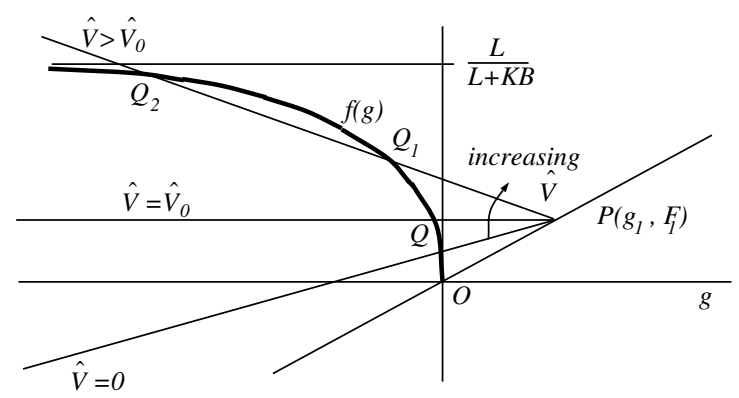

(d)

Fig. 2. (a) Graphical solution of Eqs. (38), (39) when $F_{1}<0$ and $T_{A}-T_{B}<0$. (b) Graphical solution when $F_{1}>L /(L+K B)$ and $T_{A}-T_{B}<0$. (c) Graphical solution when $0<F_{1}<L /(L+K B)$, and $T_{A}-T_{B}<0$. (d) Graphical solution when $0<F_{1}<L /(L+K B)$, and $T_{A}-T_{B}>0$.

It can be shown that the lines $F_{\mathrm{c}}(g), F_{s}(g)$ always pass through the point $P\left(g_{1}, F_{1}\right)$, where

$$
g_{1}=-\frac{2 K A\left(g_{0}-\alpha L T_{A}\right)}{\alpha L\left(T_{A}-T_{B}\right) K B-2 K A}, \quad F_{1}=-\frac{2 K B\left(g_{0}-\alpha L T_{A}\right)}{\alpha L\left(T_{A}-T_{B}\right) K B-2 K A} .
$$

This point is independent of $\widehat{V}$, so as $\widehat{V}$ is increased, the line segment $F_{\mathrm{c}}(g)$ simply rotates about the point $P$, whilst $F_{s}(g)$ remains unchanged at the slope corresponding to $\widehat{V}=0$. Notice that $F_{1}=B g_{1} / A$, so the point $P$ lies on a straight line of slope $B / A$ passing through the origin as shown.

The evolution of the intersections obtained with increasing $\widehat{V}$ and hence the qualitative behaviour of the system depends on

(1) whether the point $P$ lies in the segment $F_{1}<0,0<F_{1}<L /(L+K B)$, or $F_{1}>L /(L+K B)$, and

(2) the slope of the line segment $F_{s}(g)$.

For the case illustrated in Fig. 2(a), $F_{1}<0$ and $F_{s}(g)$ has negative slope corresponding to $T_{A}-T_{B}<0$, from (41). In this case, there is a unique stable steady-state involving contact for $\widehat{V}=0$, represented by the intersection $Q_{0}$. If $\widehat{V}$ is increased, the line segment $F_{\mathrm{c}}(g)$ rotates counterclockwise about $P$, causing the intersection to move towards increasingly negative $g$. In other words, the steady-state contact pressure 
increases with increasing speed, as we should expect. We know that this unique steady-state must also be stable, from Section 5. However, when we reach the value

$$
\widehat{V}=\widehat{V}_{0} \equiv 1+\frac{L}{K B}
$$

the line segment $F_{\mathrm{c}}(g)$ becomes horizontal and the predicted contact pressure becomes unbounded. For $\widehat{V}>\widehat{V}_{0}$, there is no intersection between $F_{\mathrm{c}}(g)$ and $f(g)$ and hence the system has no steady-state. Notice that the value $\widehat{V}_{0}$ agrees with that established in (34) for the resistance law (35).

Similar arguments can be used to predict the behaviour for other cases, involving different locations for the point $P$ and different initial slopes for the line $P Q$. Three additional cases are illustrated in Fig. 2(b-d).

Fig. 2(b) corresponds to the case $F_{1}>L /(L+K B)$ and $T_{A}-T_{B}<0$, for which there is a stable separation solution $Q_{1}$ for all values of $\widehat{V}$. This solution is unique for $\widehat{V}<\widehat{V}_{0}$, but above $\widehat{V}_{0}$ the line segment $F_{\mathrm{c}}(g)$ intersects the curve $f(g)$ defining a second (unstable) steady-state $Q_{2}$ involving contact, for which the contact pressure falls with increasing $\widehat{V}$.

Fig. 2(c) corresponds to a case $0<F_{1}<L /(L+K B)$, and $T_{A}-T_{B}<0$ in which there are three steadystate solutions at $\widehat{V}=0$, one involving separation and the other two contact. The intermediate solution $Q_{2}$ is unstable and the outer solutions $Q_{1}, Q_{3}$ are stable. Increasing $\widehat{V}$ causes the two intersections $Q_{2}, Q_{3}$ to diverge until $Q_{3}$ disappears for $\widehat{V} \geqslant \widehat{V}_{0}$.

Fig. 2(d) corresponds to the case $0<F_{1}<L /(L+K B)$, and $T_{A}-T_{B}>0$, for which there is a unique stable contact solution $Q$ for $\widehat{V}=0$. Increasing $\widehat{V}$ in this case corresponds to clockwise rotation of the line segment $F_{\mathrm{c}}(g)$, which increases the contact pressure in the steady-state. Above $\widehat{V}=\widehat{V}_{0}$, a second (unstable) steady-state $Q_{2}$ is obtained at large contact pressure, whilst $Q_{1}$ remains stable. The two intersections move towards each other with increasing $\widehat{V}$ until they merge when the line segment $F_{\mathrm{c}}(g)$ is tangential to the curve $f(g)$. We define the speed at which this tangential condition occurs as $\widehat{V}_{t}$. For $\widehat{V}>\widehat{V}_{t}$, there are no steadystate solutions.

The conclusions from these and other cases can be summarized as follows:

(i) $\boldsymbol{F}_{1}<0$ or $\boldsymbol{F}_{1}>\boldsymbol{L} /(\boldsymbol{L}+\boldsymbol{K B})$.

(a) If $g_{0}-\alpha L T_{A}>0$, the system has a stable steady-state involving separation $(g>0)$ for all values of $\widehat{V}$. This solution is unique for $\widehat{V}<\widehat{V}_{0}$, but a second solution involving contact $(g<0)$ is obtained for $\widehat{V}>\widehat{V}_{0}$ and the corresponding contact pressure falls with increasing $\widehat{V}$. This contact solution is always unstable.

(b) If $g_{0}-\alpha L T_{A}<0$, there is a unique stable contact solution for $\widehat{V}<0$ and the corresponding contact pressure increases with $\widehat{V}$, becoming unbounded at $\widehat{V}=\widehat{V}_{0}$. For $\widehat{V}>\widehat{V}_{0}$ there is no steady-state solution.

(ii) $\mathbf{0}<\boldsymbol{F}_{\mathbf{1}}<\boldsymbol{L} /(\boldsymbol{L}+\boldsymbol{K} \boldsymbol{B})$.

(a) If $g_{0}-\alpha L T_{A}>0$, the system has a stable steady-state involving separation $(g>0)$ for all values of $\widehat{V}$, but depending on the value of $T_{A}-T_{B}$ it may also have two contact solutions at $\widehat{V}=0$. If so, the lower pressure solution is unstable, the higher pressure solution is stable and these solutions move apart with increasing $\widehat{V}$, until the solution with the higher contact pressure becomes unbounded at $\widehat{V}=\widehat{V}_{0}$. For $\widehat{V}>\widehat{V}_{0}$ there is one unstable contact solution as well as the stable separation solution.

(b) If $g_{0}-\alpha L T_{A}>0$ and the separation solution is unique at $\widehat{V}=0$, there will be a value $\widehat{V}_{t}\left(<\widehat{V}_{0}\right)$ at which we obtain a double contact root, corresponding to the case where the line segment $F_{\mathrm{c}}(\mathrm{g})$ is tangent to the curve $f(g)$. For $\widehat{V}_{t}<\widehat{V}<\widehat{V}_{0}$, the roots bifurcate with increasing $\widehat{V}$, the solution with the lower contact pressure being unstable, whilst that with the higher contact pressure is stable, the corresponding contact pressure becoming unbounded at $\widehat{V}=\widehat{V}_{0}$. For $\widehat{V}>\widehat{V}_{0}$ there is one unstable contact solution as well as the stable separation solution. 
(c) If $g_{0}-\alpha L T_{A}<0$, there is a unique stable contact solution for $\widehat{V}<\widehat{V}_{0}$ and the corresponding contact pressure increases with $\widehat{V}$. For $\widehat{V}_{0}<\widehat{V}_{0}<\widehat{V}_{t}$ a second unstable contact solution is obtained for which the contact pressure decreases with increasing $\widehat{V}$. At $\widehat{V}=\widehat{V}_{t}$, the two roots merge and for $\widehat{V}>\widehat{V}_{t}$, there is no steady-state solution.

\section{Numerical simulation}

The above summary confirms that for $\widehat{V}<\widehat{V}_{0}$ there is always an odd number (one or three) of steadystate solutions, whereas for $\widehat{V}>\widehat{V}_{0}$ there are either zero or two steady states. To explore the transient behaviour of the system, a numerical model of the system was constructed. Details of the numerical algorithm are given in Appendix A. In all the examples the rod was divided into 100 finite elements of equal length.

In the first example, the parameters ${ }^{1}$ were chosen such that $F_{1}<0$ and $g_{0}-\alpha L T_{A}<0$, corresponding to case (i)b of Section 6 , for which there is a unique bounded steady-state solution with contact for $\widehat{V}<\widehat{V}_{0}$ and no steady-state solution for $\widehat{V}>\widehat{V}_{0}$.

Fig. 3 shows the evolution of the contact pressure with time at two different sliding speeds, $\widehat{V}=0.95 \widehat{V}_{0}$ and $\widehat{V}=1.05 \widehat{V}_{0}$. As initial conditions a linear distribution was used with $T_{A}(t=0)=0$ and 21 evenly spaced values of $T_{C}(t=0)$ between 0 and 1000, giving two series of 21 curves each. The results confirm that when $\widehat{V}=0.95 \widehat{V}_{0}$ the pressure tends to a unique finite value for all initial conditions, whereas when $\widehat{V}=1.05 \widehat{V}_{0}$ it grows without limit.

In the second example parameters were chosen ${ }^{2}$ to give $F_{1}>L /(L+K B)$ and $g_{0}-\alpha L T_{A}>0$, corresponding to case (i)a of Section 6. Fig. 4 shows the evolution of the temperature $T_{C}$ for $\widehat{V}>\widehat{V}_{0}$ and 51 different initial states. The results show that for low initial values of $T_{C}$, the temperature drops with time, tending eventually to the stable separation state, whereas for large initial values, the temperature and contact pressure grows without limit.

Fig. 5 shows a similar set of results where the initial conditions were chosen to be within \pm 1 temperature units of the unstable steady-state solution involving contact. Deviation from this state is initially very slow, but eventually the trend is as in Fig. 4, with the final state depending on the sign of the initial deviation from the unstable steady-state. In both Figs. 4 and 5, a change in the time derivative of $T_{C}$ is detectable at the point where the rod loses contact with the wall. This is of course associated with the corresponding discontinuity in derivative of the resistance function (35). However, any practical resistance function will involve a rapidly changing resistance near the transition from contact to separation, implying the necessity for careful time and space discretization in this range.

In the final example, parameters were chosen ${ }^{3}$ corresponding to case (ii)a of Section 6 , with two contact solutions and one separation steady-state at $\widehat{V}=0$. Fig. 6 shows the evolution of $T_{C}$ with $\widehat{V}<\widehat{V}_{0}$ and various initial conditions. For low initial values of $T_{C}$, the system evolves towards the separation steadystate, whereas for high initial values it tends to the stable contact steady-state. With these parameters, the system also has an unstable steady-state involving contact. Starting from an initial condition close to this state results in initially much slower deviation from the steady-state, but ultimately the results are similar to those shown in Fig. 6. With the same parameters but $\widehat{V}>\widehat{V}_{0}$, the stable contact solution is lost and the system evolution is qualitatively similar to that shown in Fig. 4.

\footnotetext{
${ }^{1}$ The parameters were $k=1, K=1, L=10, f=1, E=1, \alpha=2.4 \times 10^{-3}, A=1, B=100, g_{0}=-1, T_{A}=0$ and $T_{B}=1000$ in some consistent set of units. If these units are taken to be SI-units, the units of time will be seconds.

${ }^{2} k=1, K=1, L=10, f=1, E=1, \alpha=2.4 \times 10^{-3}, A=1, B=25, g_{0}=4, T_{A}=0$ and $T_{B}=1000$.

${ }^{3} k=1, K=1, L=10, f=1, E=1, \alpha=2.4 \times 10^{-3}, A=1, B=10, g_{0}=4, T_{A}=0$ and $T_{B}=1000$.
} 


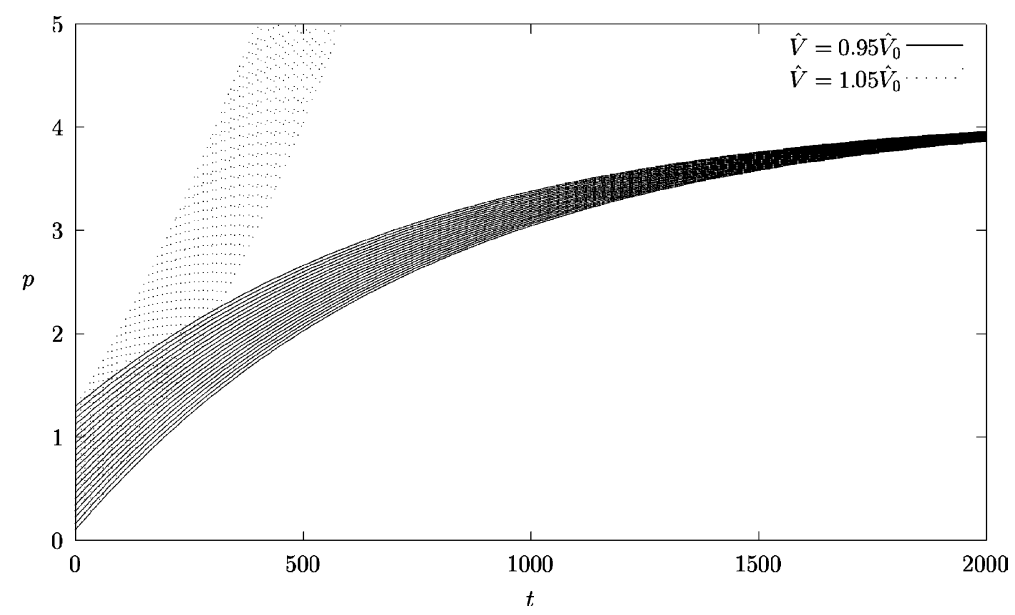

Fig. 3. Evolution of the contact pressure $p$ for an example of case (i)b.

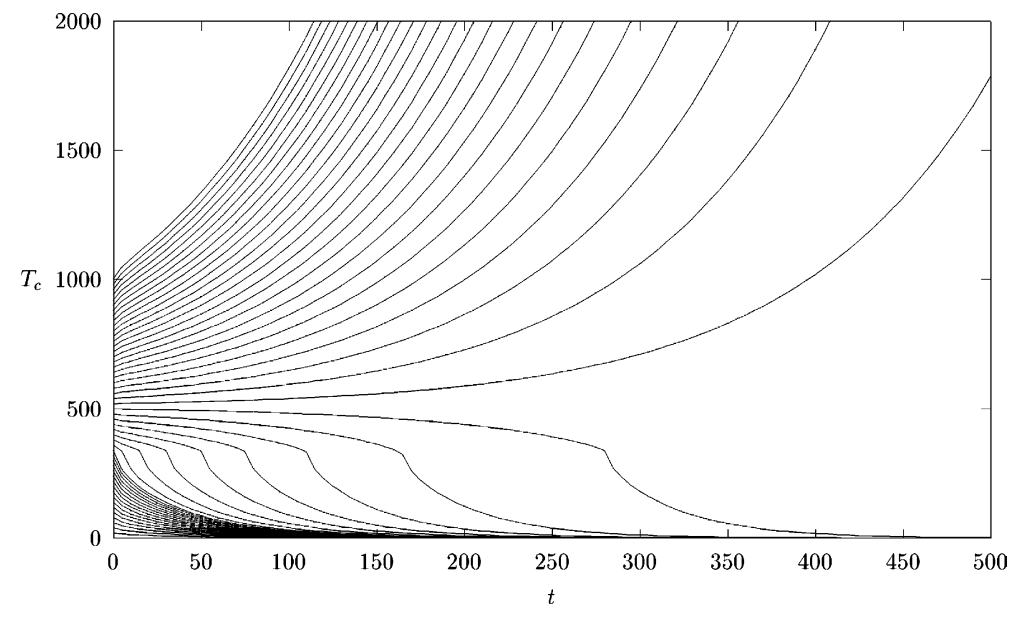

Fig. 4. Evolution of $T_{C}$ for an example of case (i)a.

\section{Discussion}

In investigations of frictionally-excited TEI, the critical speed is generally defined as that at which the solution involving full contact becomes unstable. Transient analyses of such systems typically show that the system then tends to an alternative stable steady-state involving partial contact (Zagrodzki et al., 2001).

The present results show that the concept of stability is rather more complex for the rod model. The introduction of a pressure-dependent contact resistance introduces the possibility of non-uniqueness of the steady-state even when the sliding speed $\widehat{V}=0$ and in this case at least one of the steady states is unstable. However, if $\widehat{V}<\widehat{V}_{0}$ the system always tends eventually to a stable steady-state.

For $\widehat{V}>\widehat{V}_{0}$, there are always some initial conditions for which the long time transient solution involves unbounded growth of the contact pressure. This is analogous to the phenomenon of seizure of journal bearings (Burton and Staph, 1967; Tu and Stein, 1995). However, if $g_{0}-\alpha L T_{A}>0$ there is a stable separation solution at all speeds and this will be the long time state for some initial conditions. 


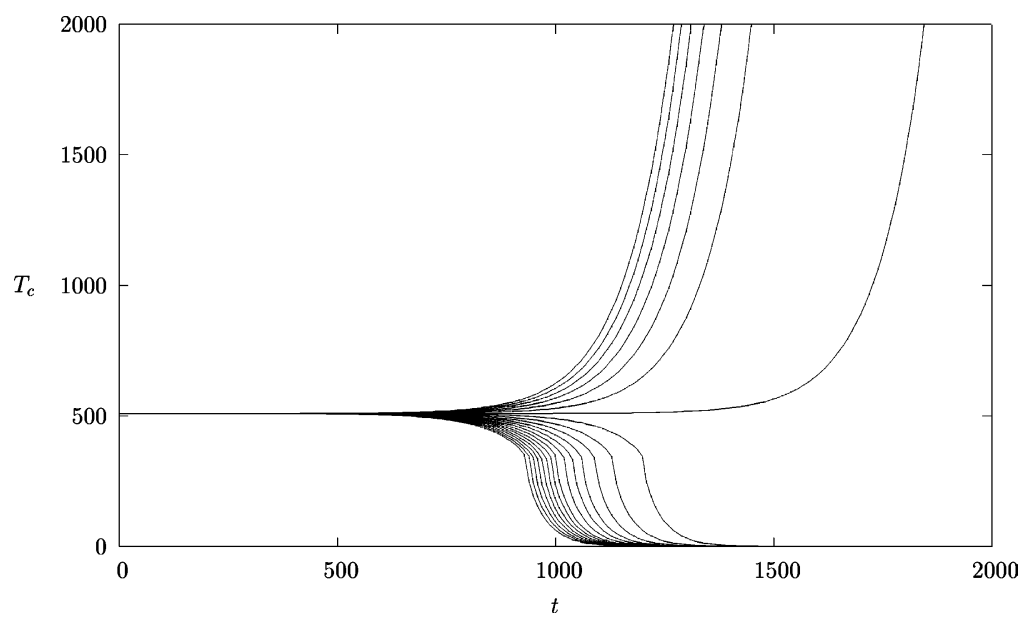

Fig. 5. Evolution of $T_{C}$ for initial conditions near to the unstable steady-state of the system of Fig. 4.

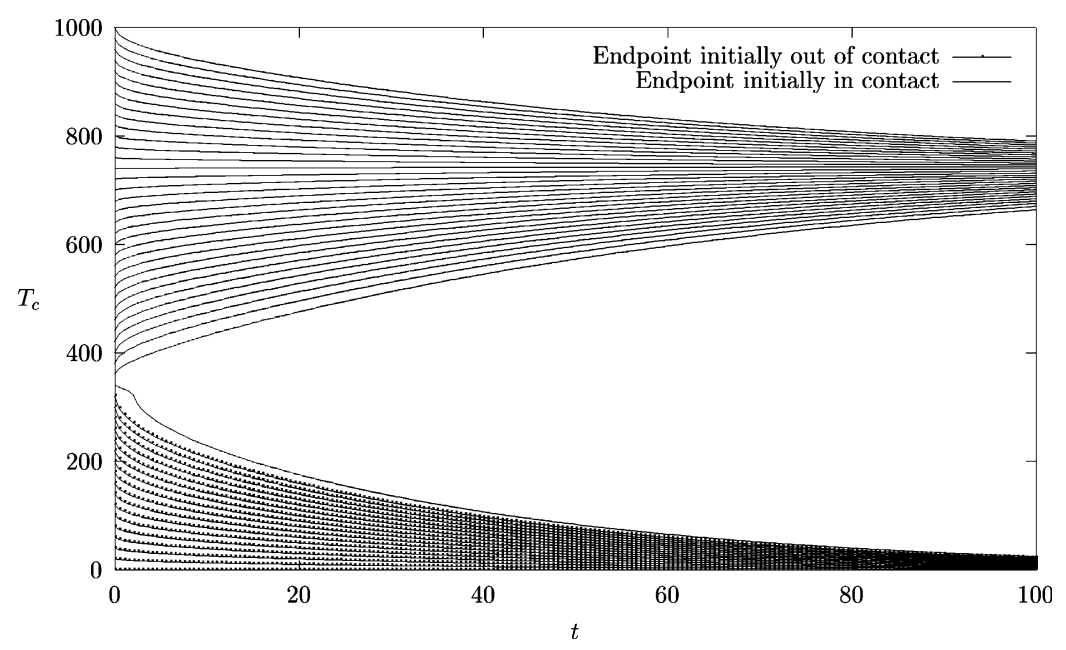

Fig. 6. Evolution of $T_{C}$ for an example of case (ii)a.

If $g_{0}-\alpha L T_{A}<0$ so that there is no separation solution, seizure will occur for all initial conditions when $\widehat{V}>\widehat{V}_{0}$, unless $0<F_{1}<L /(L+K B)$.

In the special case where $g_{0}-\alpha L T_{A}<0$ and $0<F_{1}<L /(L+K B)$, we can define a second transition speed $\widehat{V}_{t}$, corresponding to the speed at which the line segment $F_{\mathrm{c}}(g)$ is tangent to the curve $f(g)$ in Fig. 2(d). In this case, seizure will always occur when $\widehat{V}>\widehat{V}_{t}$, but depending on the initial conditions, the long time state may be either seizure or the stable contact solution $\left(Q_{1}\right.$ in Fig. 2(d)) for $\widehat{V}_{0}<\widehat{V}<\widehat{V}_{t}$.

\section{Conclusions}

In the present paper, we have investigated the interaction between frictional heating and heat conduction across a pressure-dependent thermal contact resistance at a contact interface in the idealized problem of a 
thermoelastic rod contacting a rigid wall at prescribed temperature. For sufficiently low sliding speeds $\left(\widehat{V}<\widehat{V}_{0}\right)$, the results are qualitatively similar to those with no sliding. In particular, the number of steadystate solutions is odd; if the steady-state is unique it is stable and if it is non-unique, stable and unstable solutions alternate, with the outlying solutions being stable. By contrast, for $\widehat{V}>\widehat{V}_{0}$ either there are no steady-state solutions (non-existence of solution) or the number of steady states is even. In the latter case, stable and unstable states again alternate, so that there is always an outlying unstable steady-state. Notice that the limiting speed $\widehat{V}_{0}$ is defined by Eq. (34) and depends only on the properties of the rod and the limiting value of contact resistance as contact pressure $p \rightarrow \infty$.

A numerical study for the special case where the resistance function is defined by Eq. (35) shows that when the system has no steady states, the contact pressure grows without limit from any initial condition. If it has steady states, but $\widehat{V}>\widehat{V}_{0}$, the system will either tend to a stable steady-state or the contact pressure will increase without limit, depending on the initial condition. In all cases, if $V<V_{0}$ the system will tend to one of the stable steady states.

\section{Appendix A}

In this section the numerical method used for the examples in Section 7 above is outlined. In this method the entire problem is solved (after discretization) as a system of (non-smooth) equations at each timestep using a direct application of Newton's method.

The equations to be solved are

$$
\begin{aligned}
& \frac{\partial^{2} T}{\partial x^{2}}-\frac{1}{k} \frac{\partial T}{\partial t}=0, \quad 0<x<L, \\
& K \frac{\partial T}{\partial x}+\frac{1}{2}(1-\operatorname{sign}(g))\left[\frac{g}{B g-A}\left(T_{C}-T_{B}\right)+\frac{f V g E}{L}\right]=0, \quad x=L, \\
& g-g_{0}+\alpha \int_{0}^{L} T \mathrm{~d} x=0,
\end{aligned}
$$

where $\operatorname{sign}(g)=1$ if $g \geqslant 0$ and $\operatorname{sign}(g)=-1$ if $g<0$. The temperature at $x=0$, the temperature $T_{B}$ of the moving wall, the initial gap (or pressure) $g_{0}$ and the initial temperature distribution are prescribed. In the actual implementation a dimensionless form of the equations was used.

For the discretization, the rod is divided into $n+1$ finite elements so that there are $n$ internal nodes. A sequence of times $\left[t^{\text {tart }}, \ldots, t^{k}, t^{k+1}, \ldots, t^{\text {final }}\right]$ is introduced. Eqs. (A.1)-(A.3) will be discretized to form a system of $n+2$ non-linear equations for the $n$ temperatures at the internal nodes, the temperature at $x=L$ and $g$, all at time $t^{k+1}$ and assuming that everything is known at time $t^{k}$. In what follows, $T_{i}^{k}$ is the temperature at spatial node $i, T_{C}^{k}$ is the temperature at $x=L$ and $g^{k}$ is the value of $g$, at time $t^{k}$.

First, discretizing Eq. (A.1) by linear finite elements in the spatial coordinate and an Euler type backward finite difference in time we obtain at each internal node

$$
\begin{aligned}
f_{i}= & \left(\frac{1}{h_{i+1}}-\frac{c h_{i+1}}{2}\right) T_{i+1}^{k+1}-\left(\frac{1}{h_{i+1}}+\frac{1}{h_{i-1}}+c h_{i+1}+c h_{i-1}\right) T_{i}^{k+1}+\left(\frac{1}{h_{i-1}}-\frac{c h_{i-1}}{2}\right) T_{i-1}^{k+1} \\
& +\frac{c h_{i+1}}{2} T_{i-1}^{k}+\left(c h_{i+1}+c h_{i-1}\right) T_{i}^{k}+\frac{c h_{i-1}}{2} T_{i-1}^{k}=0 \quad i=1, \ldots, n,
\end{aligned}
$$

where $h_{i+1}$ and $h_{i-1}$ are the length of the elements to the left and right of node $i$, respectively and $c=1 / 3 k\left(t^{k+1}-t^{k}\right)$. 
Next, Eq. (A.2) is discretized using a four-point finite difference approximation for $\partial T / \partial x$ since it was suspected, and confirmed by some numerical experiments, that this would improve the accuracy compared to using a simple two point formula. Thus

$$
\begin{gathered}
f_{n+1}=11 / 6 T_{C}^{k+1}-3 T_{n}^{k+1}+3 / 2 T_{n-1}^{k+1}-1 / 3 T_{n-2}^{k+1}+\frac{1}{2}\left(1-\operatorname{sign}\left(g^{k+1}\right)\right)\left[\frac{\Delta h g^{k+1}}{B g^{k+1}-A}\left(T_{C}^{k+1}-T_{B}^{k+1}\right)\right. \\
\left.+\frac{\Delta h f V g^{k+1} E}{L}\right]=0,
\end{gathered}
$$

where $\Delta h$ is the length of the four elements at the right end of the rod; these elements are assumed to have equal length for the sake of this discretization.

Finally, Eq. (A.3) is discretized as

$$
f_{n+2}=g^{k+1}-g_{0}+\alpha \sum_{i=0}^{n+1} T_{i}^{k+1} w_{i}=0,
$$

where the $w_{i}$ are appropriate integration weights.

Eqs. (A.4)-(A.6) form a system of $2 n+2$ nonlinear equations for the $n+2$ unknowns $T_{i}^{k+1}, T_{C}^{k+1}$ and $g^{k+1}$. Putting

$$
\boldsymbol{f}(\boldsymbol{z})=\left(f_{1}, \ldots, f_{i}, \ldots, f_{n}, f_{n+1}, f_{n+2}\right)^{t}
$$

and

$$
\boldsymbol{z}=\left(T_{1}^{k+1}, \ldots, T_{i}^{k+1}, \ldots, T_{n}^{k+1}, T_{C}^{k+1}, g^{k+1}\right)^{t}
$$

the solution is found by a direct application of Newton's method, that is, finding a solution to $\boldsymbol{f}(\boldsymbol{z})=\mathbf{0}$ by applying

$$
\begin{aligned}
& -\frac{\partial \boldsymbol{f}}{\partial \boldsymbol{z}_{j}} \Delta \boldsymbol{z}_{j}=\boldsymbol{K} \Delta \boldsymbol{z}_{j}=\boldsymbol{f}\left(\boldsymbol{z}_{j}\right) \\
& \boldsymbol{z}_{j+1}=\boldsymbol{z}_{j}+\Delta \boldsymbol{z}_{j}
\end{aligned}
$$

repeatedly until $\|\boldsymbol{f}\|_{2}<$ tol. Here a subscript $j$ refers to the Newton iterations. The derivatives of $f_{i}$ and $f_{n+2}$ needed to form $\boldsymbol{K}$ are easily computed. Equation $f_{n+1}$ is, unfortunately, not differentiable in the usual sense, but it has the property of being B-differentiable, meaning essentially that directional derivatives exist at each point. This makes it possible to apply the Newton method deviced for such equations by Pang (1990). Here, a somewhat simplified version of this method was used where the necessary derivatives were computed by arbitrarily picking one such directional derivative at non-differentiable points, see Christensen et al. (1998). In the present implementation the following derivatives of $f_{n+1}$ with respect to $T_{C}^{k+1}$ and $g^{k+1}$ were used

$$
\begin{aligned}
& \frac{\partial f_{n+1}}{\partial T_{C}^{k+1}}= \begin{cases}\frac{11}{6}+\frac{\Delta h g^{k+1}}{B g^{k+1}-A} & g<0 \\
\frac{11}{6} & g \geqslant 0\end{cases} \\
& \frac{\partial f_{n+1}}{\partial g^{k+1}}= \begin{cases}\left(\frac{\Delta h}{B g^{k+1}-A}-\frac{B \Delta h g^{k+1}}{\left(B g^{k+1}-A\right)^{2}}\right)\left(T_{C}^{k+1}-T_{B}^{k+1}\right)+\frac{\Delta h f V g E}{L} & g<0 \\
0 & g \geqslant 0\end{cases}
\end{aligned}
$$

while the remaining derivatives are easily computed. In most of the numerical computations Newton's method was applied with the linesearch method suggested by Pang (1990). 


\section{References}

Barber, J.R., 1967. The distribution of heat between sliding surfaces. J. Mech. Engng. Sci. 9, 351-354.

Barber, J.R., 1969. Thermoelastic instabilities in the sliding of conforming solids. Proc. Roy. Soc. A 312, $381-394$.

Barber, J.R., 1970. The conduction of heat from sliding solids. Int. J. Heat Mass Transfer 13, 857-869.

Barber, J.R., Dundurs, J., Comninou, M., 1980. Stability considerations in thermoelastic contact. ASME J. Appl. Mech. 47, 871-874.

Barber, J.R., 1986. Non-uniqueness and stability for heat conduction through a duplex heat exchanger tube. J. Thermal Stresses 9 , 69-78.

Burton, R.A., Kilaparti, S.R., Nerlikar, V., 1973. A limiting stationary configuration with partially contacting surfaces. Wear 24, 199206.

Burton, R.A., Staph, H.E., 1967. Thermally activated seizure of angular contact bearings. ASLE Trans. 10, $408-417$.

Christensen, P.W., Klarbring, A., Pang, J.S., Strömberg, N., 1998. Formulation and comparison of algorithms for frictional contact problems. Int. J. Numer. Meth. Engng. 42, 145-173.

Cooper, M.G., Mikic, B.B., Yovanovich, M.M., 1969. Thermal contact conductance. Int. J. Heat Mass Transfer 12, $279-300$.

Dow, T.A., Burton, R.A., 1972. Thermoelastic instability of sliding contact in the absence of wear. Wear 19, 315-328.

Johansson, L., 1993. Model and numerical algorithm for sliding contact between two elastic half-planes with frictional heat generation and wear. Wear 160, 77-93.

Pang, J.S., 1990. Newton's method for B-differentiable equations. Math. Oper. Res. 15, 311-341.

Shlykov, Yu.P., Ganin, Ye.A., 1964. Thermal resistance of metallic contacts. Int. J. Heat Mass Transfer 7, 921-929.

Srinivasan, M.G., France, D.M., 1985. Non-uniqueness in steady-state heat transfer in prestressed duplex tubes-Analysis and case history. ASME J. Appl. Mech. 52, 257-262.

Tu, J.F., Stein, J.L., 1995. Online preload monitoring for antifriction bearings of high speed machine tools. ASME J. Dyn. Syst. 117, 43-53.

Yeo, T., Barber, J.R., 1994. Finite element analysis of thermoelastic contact stability. ASME J. Appl. Mech. 61, 919-922.

Zagrodzki, P., Lam, K.B., Al-Bahkali, E., Barber, J.R., 2001. Nonlinear transient behaviour of a sliding system with frictionally excited thermoelastic instability. ASME J. Tribol. 123, 699-708.

Zhang, R., Barber, J.R., 1990. Effect of material properties on the stability of static thermoelastic contact. ASME J. Appl. Mech. 57, 365-369.

Zhang, R., Barber, J.R., 1993. Transient thermo-elastic contact and stability of two thin-walled cylinders. J. Thermal Stresses 16, $31-54$. 